\section{ACCURACY OF ELECTRONIC APEX LOCATOR FOR DETERMINING THE ROOT CANAL LENGTH IN PRESENCE OF BLOOD - AN IN VITRO STUDY}

A.K.M. Bashar ${ }^{1}$, Reema Joshi ${ }^{2}$, M.S. Alam ${ }^{3}$

Abstract:

Present study was conducted to evaluate the accuracy of Electronic Apex Locator to measure the root canal length in presence of blood, an unavoidable event during extirpation of vital pulp. A number of sixty (60) previously untreated extracted human maxillary and mandibular permanent mature anterior teeth having more or less straight roots with single root canal were included in the study. Root canal lengths were measured using a no. 15 k-file (Mani Inc, Tokyo Japan) in all the teeth until it was just visible through apical foramen that was confirmed by viewing them in Stereomicroscope. The measurements obtained by the Stereomicroscope of all teeth were consider as the 'Gold standard' and was also consider as the actual length of those teeth. Using an Electronic Apex locator (Foramatron D 10, Percell, USA) was used to measure the canal length of the same sixty teeth introducing human blood into the canal. Now the measurements taken by Electronic Apex locator (EAL) in presence of blood were compared with the actual length taken by the stereomicroscope and difference in the error length compared with stereomicroscopic length were calculated in a range from $-0.5 \mathrm{~mm}$ to $>1 \mathrm{~mm}$. It was observed that working length measurements by EAL in presence of blood within canal mostly remain within clinically acceptable range $( \pm 0.5 \mathrm{~mm})$.

\section{Introduction:}

Determining root canal length with accuracy is a crucial factor for success of root canal therapy. Errors in determining root canal length result in instrumentation short of the canal terminus, allowing pulp tissue and necrotic debris to remain in the canal. Faulty working length can also lead to instrumentation longer than the canal terminus thus destroying the delicate apical region. The literature suggests that the preparation and obturation of the root canal should be at or short of the apical constriction. ${ }^{1}$ To attain this objective the endpoint of the root canal system and the canal terminus, should be detected as precisely as possible during preparation of the canal.

1. Assistant Professor, Dept of Conservative Dentistry and Endodontics, Banghabandhu Sheikh Mujib Medical University, Dhaka

2. MS Part B Student, Dept of Conservative Dentistry and Endodontics, Banghabandhu Sheikh Mujib Medical University, Dhaka

3. Professor, Dept of Conservative Dentistry and Endodontics, Banghabandhu Sheikh Mujib Medical University, Dhaka
One of the innovations in root canal treatment is the development of Electronic apex locators (EAL), an electronic device for detecting the canal terminus. All electronic apex locators flowed the current from the device and establish a circuit through the two electrodes in the mouth, one electrode attached to the file and another electrode ground in the lip of the patient, the current flow back from the circuit to the device that would indicate or display in the monitor where the file was in the canal or it reached to the apex. ${ }^{2}$ It eliminates many of the problems associated with radiographic methods and it attempts to measure the length of the root canal either to the end of apical constriction or at apical foramen. It is more accurate, easy and fast, with no requirements of X-ray exposures. ${ }^{3}$

But, many of the acquisition, being made to EALs in its accuracy due to shortcomings like biological phenomena such as vital tissue, exudates and blood that create the probability of early electric circuit formation before reaching the apex. 4 Since root canal treatment many often is performed in different pulpal pathosis where the pulp remain vital; its extirpation causes bleeding in the root canal. So it must be ensured that electronic apex locator is not influenced while determining working length in presence of blood. But, the influence of presence of blood on the efficiency and accuracy of EAL has not been reported yet. The main objective of the study is to evaluate the accuracy of EAL in tooth length determination in presence of blood within the canal.

\section{Materials and method:}

This prospective in vitro study was conducted in the Department of Conservative Dentistry \& Endodontics, Bangabandhu Sheikh Mujib Medical University and partly in the Department of Geology, University of Dhaka within the period from January 2006 to December 2007. A number of sixty (60) previously untreated extracted human maxillary and mandibular permanent mature anterior teeth having more or less straight roots with single root canal (Type I), as determined by radiography, were selected as the study specimen. On the other hand teeth those fractured in root position during extraction, having open apex, radiographic disclosure of being affected by either internal or external resorption or canal blocked by calcification, all were excluded from the study. All the selected 60 teeth were immersed in 1\% sodium hypochlorite solution in a screw capped glass vial for 24 hours to remove any organic debris from the surface followed by cleaning with hand and ultra-sonic scalar to remove calculus, tartar, stain and remained organic tissue debris. Now, all the teeth were again immersed in distilled water for 24 hours prior to tooth preparation. The pulp chambers were accessed with a 701 or 702 tapered fissure bur or (straight fissure bur) in an accelerated speed contra angle handpiece with proper cooling arrangement. The roofs 
of the pulp chambers were removed with no. 2 or 4 round burs running at slow speed in contra angle micro-motor handpiece. The canal orifices were located by an endodontic explorer. Any residual pulp tissue was removed from the root canals with a barbed broach. Any irregularities, if present on incisal edges of the teeth were then, trimmed by straight fissure bur to have the univocal reference point. The final procedures were held in two phases:-

\section{Phase One}

All Sixty teeth were measured using a no. 15 k-file (Mani Inc, Tokyo Japan) in all the teeth until it was just visible through apical foramen that was confirmed by viewing them in Stereomicroscope (Meiji Techno. Co. Ltd. Tokyo, Japan) at X14 magnification and photographed for recording (Figure 1). The rubber stopper was adjusted to the level chosen as reference point for root canal measurement. An Endodontic Gauge (GC Corporation Tokyo, Japan) of $0.5 \mathrm{~mm}$ accuracy measurement was used to measure the distance from the stopper to the file tip. The measurements obtained by the Stereomicroscope of all teeth were consider as the 'Gold standard' and was also consider as the actual length of those teeth to which all other measurements were compared. All the teeth were mounted in the alginate bases separately. When not in use the models were wrapped with the damped cotton and refrigerated. The teeth with alginate bases were kept in a moist environment throughout the study period.

\section{Phase Two}

$5 \mathrm{ml}$ of blood was collected from the brachial artery and preserved in a test tube containing anti coagulant. Collected blood was slowly injected into the root canal of all the 60 teeth consecutively by using a $3 \mathrm{ml}$ disposable syringe (JMI-Bangla Co. Ltd.). Any blood overflowed to the pulp chambers was soaked with small cotton ball. Now working length measurements were taken by EAL according to the manufacturer's instruction.

During electronic measurement by Electronic apex locator (Foramatron D10, Parkell Electronic Division, Farmingdale, New York, USA), the cord with labial clip of the corresponding apex locator was inserted into the middle of the alginate base. The remaining cord of electronic apex locator with a hook was latched into the ISO 15 no. K-file (Mani Inc, Tokyo Japan). It was ensured that the hook of the EAL was in proper contact with the file. Now the $15 \mathrm{~K}$ file was slowly inserted into the blood filled root canal and observed for the electronic display. When the tip of the file approaches the foramen, the graphical display suddenly illuminated. Starting with green lights and tracked down slowly. After the progression of file down the canal, the lights switched to yellow which meant to be close to the apical area. When the tracking light flashed to red that was labeled in the display meter as 'apex', this meant to be at the apical foramen according to the manufacture's claim. (Figure 2) The measurements were taken up to that mark and noted down in the data sheets. If the file tip penetrated the apex, orange tracking light illuminated which meant beyond the foramen so the file slightly withdrawn until it reached to the targeted length. In supplement of the visual display, the device also warned with four distinct audio signals. In this way, all the measurement of sixty teeth in presence of blood within the canal was taken and noted down in data sheets. Each measurement was repeated three times and the mean value computed.

\section{Results:}

The present study was undertaken on 60 extracted teeth to evaluate the influence of presence of blood within the root canal on the accuracy of electronic apex locator in estimating length of root canal. Stereomicroscopic measurements were considered as the actual length of the teeth with which measurements taken in presence of blood were compared. Difference in the error length compared with stereomicroscopic length were calculated and distributed in different range. Lowest difference was $-0.5 \mathrm{~mm}$ and highest were $>1 \mathrm{~mm}$.

Data and comparison as obtained in the study was shown in tables:

Table-I: Tooth length measurement in different mode $(n=60)$.

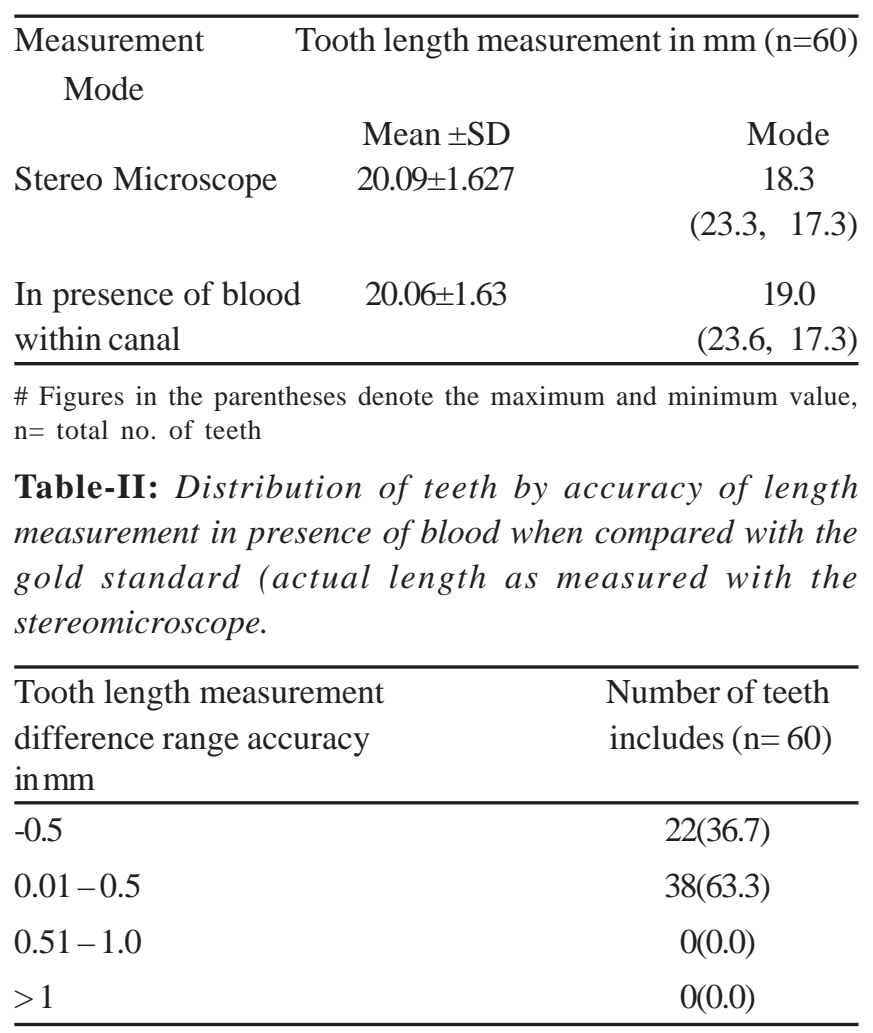


The difference with the actual length as measured in presence of blood when found between -0.5 to 0.0 were placed on that range and similarly all other teeth were distributed. Working length measurements in presence of blood within canal mostly remain within clinically acceptable range $( \pm 0.5 \mathrm{~mm})$.

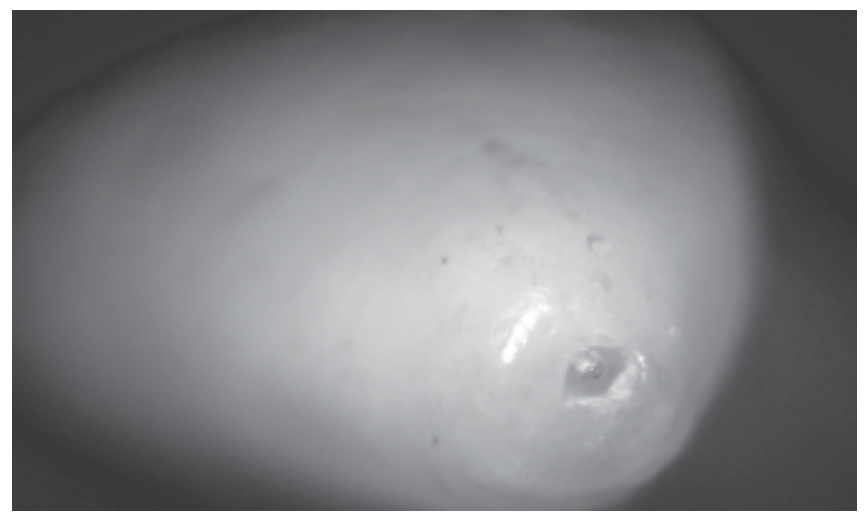

Fig.-1: Canal length measurement taken by stereomicroscope. Tip of the fine file is just visible through the apical foramen.

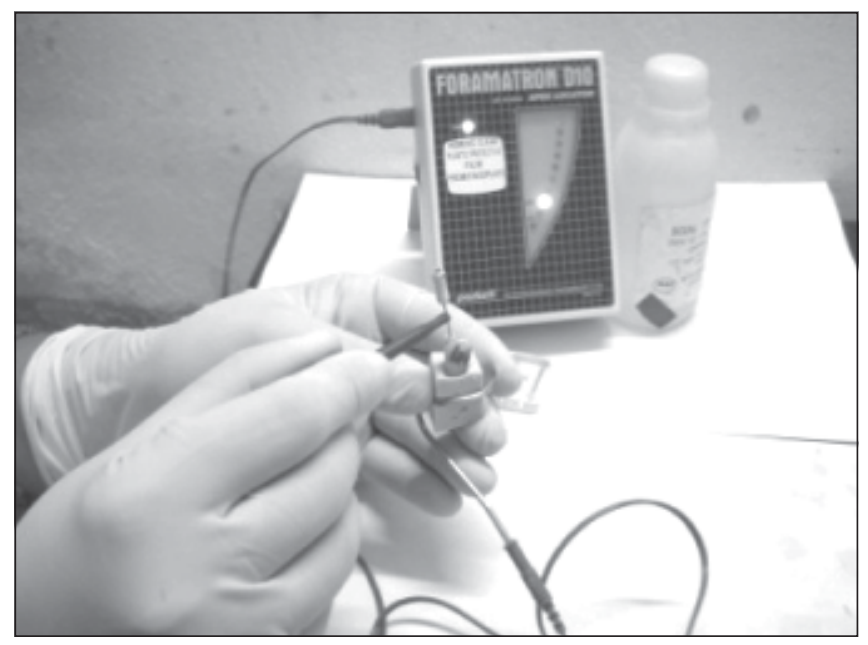

Fig.-2: Canal length measurement in presence of blood with Foramatron D 10 Electronic apex locator. Lip hook is attached to the middle of the alginate base and the other clip is firmly attached with the fine file. Now the file is inserted within the blood filled canal. Tracking light flashed to red that was labeled in the display meter as 'apex', this meant to be at the apical foramen.

\section{Discussion:}

Clinically, determination of working length for preparation and obturation play a vital role in the success of endodontics which should be at or short of apical constriction and it usually lie 0.5 to $0.8 \mathrm{~mm}$ from the major diameter or apical foramen. ${ }^{5}$ Most manufactures of modern EALs in their reports suggested to subtract $0.5 \mathrm{~mm}$ from the length of the file at the point when the device suggest the 0.0 reading or display apex to obtain the apical constriction or the most suitable working length of the root canal. ${ }^{2}$ Lee et al ${ }^{6}$ reported in their study that most of the file tips ended at the major foramen regardless of the existence of a detectable CDJ suggesting that the major foramen was more reproducible compared with the CDJ, for accuracy studies of EAL. In the current study, the reference point was the length as measured by introducing fine file tip until it was just at the level of the apical foramen confirmed by the stereomicroscope, which was taken as the actual length and the 'gold standard'. Comparison was made with the measurement of EAL when it indicated the apex (major apical foramen) and illuminated the red light in display meter.

Many of the studies used an error range of $\pm 0.5 \mathrm{~mm}$ to assess the accuracy of the EALs. ${ }^{7}$ Measurements attained within this tolerance were considered highly accurate. In comparison to these standards, the measurements of the EAL if taken within $\pm 0.5 \mathrm{~mm}$ to the foramen as clinically acceptable tolerance range, the accuracy of the present study was $100 \%$ in presence of blood within the canal. Other vitro studies, under taken on different type of the frequency dependant EALs within \pm 0.5 mm showed from 90-100\% accuracy. ${ }^{8,9,10,11}$ However, some other follow strict rule of the error tolerance and deny the consideration of $\pm 0.5 \mathrm{~mm}$ tolerance. ${ }^{12}$ Dunlup et al concluded that consideration should be given to using -0.5 to $0.0 \mathrm{~mm}$ as the most clinically ideal error tolerance range. ${ }^{13}$ If this was to be taken in consideration then in presence of blood 22 out of 60 teeth fell into the -0.5 to $0.0 \mathrm{~mm}$ as the ideal clinically tolerance. However, many of the root canals do not always end with an apical constriction, a well-delineated minor or major apical diameter, or an apical foramen within the base of the cemental cone. With a lack of such demarcations, an error tolerance upto $\pm 1.0 \mathrm{~mm}$ to the foramen is deemed to the clinically acceptable range of tolerance. ${ }^{14}$ Other studies relied on a more lax clinical range of $\pm 1.0 \mathrm{~mm}$ to the foramen. ${ }^{15}$ One reason cited for accepting a $\pm 1.0 \mathrm{~mm}$ margin of error is the wide range seen in the shape of the apical zone and root canal do not always end with an apical constriction, a clear minor and major diameter or an apical foramen at the exact base of the cemental zone. ${ }^{14}$ With this consideration Electronic apex locator in presence of blood within the canal, according to present study, gave $100 \%$ accuracy within $\pm 1 \mathrm{~mm}$ clinically acceptable range of tolerance.

Results of an in vitro study may raise doubts about its clinical relevance. The validity of in vitro models to study the accuracy of EALs operates on principles of electrictricity rather than biological properties of the tissue involved. Therefore, in vitro models in which extracted teeth were immersed in media with similar electrical resistance to the periodontium can provide valuable information into the function of EALs. Researchers have found alginate, gelatine, agar or saline to be media that 
give predictable results with apex locators when compared with tooth length. ${ }^{8,16}$ Subramaniam et al used the alginate mould prepared with $0.9 \%$ of $\mathrm{NaCl}$ and concluded that it simulated the periodontium. ${ }^{17}$ This solution had become a benchmark since its ionic content is equivalent to that of blood plasma and biological tissue simulates the $0.9 \% \mathrm{NaCl}{ }^{18}$ Kaufman et al. used these experimental models for 45 days preserving them in moist environment like in refrigerator and said that such environment is satisfactory. ${ }^{19,20}$ The alginate model with $0.9 \% \mathrm{NaCl}$ used in this study and preserved in refrigerator during study period was found to be accurate, easy to use and reliable.

\section{Conclusion:}

In the light of the finding of the study conclusions can be made that Electronic Apex Locator (EAL) can measure working length with $100 \%$ accuracy within $\pm 0.5 \mathrm{~mm}$ range of absolute errors, which is clinically acceptable. But comparative studies assessed by radiographs, and the validity of the results is open to question.

\section{References:}

1. Gordon MPJ, Chandler NP. Electronic apex locators. Int Endod J 2004; 37:1-13.

2. Nekoofar MH, Ghandi MM, Hayes SJ, Dummer PMH. The fundamental operating principles of electronic root canal length measurement devices. Int Endod J 2006;39:595-609.

3. Kobayashi C. Electronic canal length measurement. Oral Sur,Oral Med, Oral Path, Oral Rad. 1995; 79:226-31.

4. Trope M, Rabie G, Tronstad L. Accuracy of an electronic apex locator under controlled clinical conditions. Endod Dent Traumatol 1985;1:142-5.

5. Ricucci D, Langeland K. Apical limit of root canal instrumentation and obturation part 2. A histological. Int Endod J 1998; 31:394-409.

6. Lee SJ, Nam KC, Kim YJ, Kim DW. Clinical accuracy of a new apex locator with an automatic compensation circuit. J Endod. 2002; 28: 706-9.

7. Pagavino G, Pace R, Baccetti T. A SEM study of in vivo accuracy of the Root ZX electronic apex locator. J Endod.1998; 24:438-41.
8. Czerw RJ, Fulkerson MS, Donnelly JC. An in vitro test of a simplified model to demonstrate the operation of electronic root canal measuring devices. J Endod 1994; 20:605-6.

9. De Moor RJG, Hommez GMG, Martens LC, De Boever JG. Accuracy of four electronic apex locators: an in vitro evaluation. Endod Dent Traumatol. 1999;15:77-82.

10. Felippe MC, Soares IJ. In vitro evaluation of an audiometric device in locating the apical foramen of teeth conditions. Endod DentTraumatol.1994;10:220-2.

11. Frank AL, Torabinejad M. An in vivo evaluation of Endex electronic apex locator. J Endod 1993; 19:177-9.

12. Kaufman AY, Keila S, Yoshpe M. Accuracy of a new apex locator: an in vitro study. Int Endod J 2002; 35: 186-92.

13. Dunlap C, Remeikis N, BeGole E, Rauschenberger C. An in vivo evaluation of an electronic apex locator that uses the ratio method in vital and necrotic canals. J Endod, 1998; 24: 48-50.

14. Shabahang S, Goon W, Gluskin A. An in vivo evaluation of Root ZX electronic apex locator. J Endod 1996;22:616-8.

15. Keller ME, Brown CE Jr, Newton CW. A clinical evaluation of the Endocater-an electronic apex locator. J Endod 1991; $17: 271-4$.

16. Meares WA, Steiman HR. The influence of sodium hypochlorite irrigation on the accuracy of the Root ZX electronic apex locator. J Endod 2002; 28:595-8.

17. Subramaniam P, Konde S, Mandanna DK. An in vitro comparison of root canal measurement in primary teeth. $J$ IndianSoc Pedod Prev Dent 2005; 9: 124-1.

18. Pallas-Areny R, Baccetti T. A SEM study of in vivo accuracy of the Root ZX electronic apex locator. J Endod 1998; 40:830-3.

19. Nguyen HQ, Kaufman AY, Komorowski RC, Friedman S. Electronic length measurement using small and large files in enlarged canals. Int Endod J 1996; 29:359-64.

20. Fuss Z, Assooline LS, Kaufman AY. Determination of location of root perforations by electronic apex locators. Oral Sur Oral Med Oral Path Oral Rad Endod 1996; 82: 324-9. 